\title{
Unexpected ring contraction of 1-aryl-cyclohexa-2,5-dienes under palladium catalysis
}

\author{
Raphaël Lebeuf, Frédéric Robert, and Yannick Landais* \\ Université de Bordeaux, Institut des Sciences Moléculaires, UMR-CNRS 5255,351, cours de la \\ libération, 33405 Talence, Cedex, France \\ E-mail:y.landais@ism.u-bordeaux1.fr
}

\section{Dedicated to Prof. Pierre Vogel on the occasion of his $70^{\text {th }}$ anniversary}

DOI: http://dx.doi.org/10.3998/ark.5550190.p008.241

\begin{abstract}
The reaction of 1,1-disubstituted cyclohexa-2,5-dienes with aryl iodides in the presence of $\operatorname{Pd}(0)$ unexpectedly provided bicyclo[3.1.0] compounds in a very diastereocontrolled fashion in moderate to good yield. A tentative mechanism is proposed to rationalize this unusual reactivity of cyclohexadienes under palladium catalysis.
\end{abstract}

Keywords: Catalysis, Heck reaction, Wacker oxidation, palladium, cyclohexadienes, ring contraction

\section{Introduction}

1-Substituted cyclohexa-2,5-dienes constitute a versatile family of building blocks which continue to attract a wide interest. ${ }^{1-4}$ Desymmetrization of such dienes has been carried out through a number of ways, leading to advanced intermediates in the synthesis of natural products. In the meantime, 1-silylcyclohexa-2,5-dienes and cyclohexa-2,5-diene carboxylic acids were shown to be valuable surrogate of tin hydride in radical processes. ${ }^{4}$ Our continuous interest for this class of compounds $s^{1,5,6}$ has led recently to the development of a methodology focused on a palladium-catalyzed aza-Wacker process $^{7-9}$ using 1 -arylcyclohexa-2,5-dienes as precursors. ${ }^{10}$ The pendant ethylamino moiety on the quaternary center was thus shown to add onto the dienyl system, affording the corresponding 5-membered ring along with a new conjugated diene, which could be functionalized further. For instance, starting from an ortho-substituted arylcyclohexa2,5-diene such as $\mathbf{1}$, this strategy offered a straightforward entry toward the tetracyclic core 2 of aspidosperma alkaloids (Scheme 1). Reoxidation of $\operatorname{Pd}(0)$ into $\operatorname{Pd}(\mathrm{II})$ is carried out using a stream of oxygen, the charcoal serving as to disperse the palladium catalyst, avoiding the 
formation of metal colloids which precipitate during the reaction, thus limiting catalyst turn-over. Moderate to good yields of the cyclized product were thus observed using this protocol.
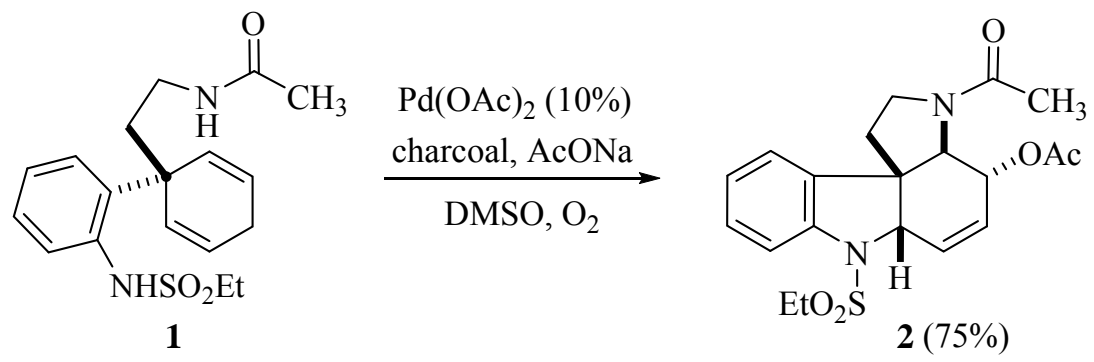

Scheme 1. Pd(II)-catalyzed aza-Wacker cascade.

The slow $\mathrm{Pd}(0) \rightarrow \mathrm{Pd}(\mathrm{II})$ reoxidation by oxygen (also producing hydrogen peroxide) may be circumvented using a $\mathrm{Pd}(0)$ source in the presence of an haloalkane (1,2-dichlorethane (DCE) or chlorobenzene), which upon oxidative addition generates a Pd(II) species. This strategy was used by Guram and $\mathrm{Bei}^{11,12}$ for the oxidation of alcohols into ketones using a $\operatorname{Pd}(0)$ catalyst. Interestingly, this protocol is compatible with substrates bearing an olefinic moiety. Application of this strategy to our case was envisaged having in mind the possible competition between this oxidation and intermolecular Heck reaction, following a pathway depicted in Figure 1 below. A Heck reaction-heterocyclization could effectively take place after the regioselective arylpalladation of the diene. ${ }^{13-17}$ The regioselectivity of the process would be directed by the ethylamino group as in $\mathbf{I}$, which upon reductive elimination would give rise to the bicyclic system II (Figure 1). Mechanisms have recently been put forward by Wolfe for such heterocyclizations, assuming the formation of a Pd-heteroatom bond preceding the cyclization, the C-Ar bond being generated through reductive elimination. ${ }^{18-19}$ Based on these premises, we started a study on the reactivity of arylcyclohexadienes $\mathbf{3}$ with aryl iodides $\mathbf{4}$ in the presence of a $\operatorname{Pd}($ II) catalyst. We report herein the addition of aryl halides onto dienes $\mathbf{3}$ and the unusual reactivity of the latter, which afford under these conditions cyclopropanes 5. A tentative mechanism is provided to explain the unexpected formation of 5. 




Figure 1. Intermolecular Heck reaction with 1-arylcyclohexa-2,5-dienes.

\section{Results and Discussion}

Cyclohexadienes 3a-d were prepared through a Birch reductive alkylation using $\alpha$-chloronitrile as electrophile, followed by the reduction of the nitrile into an amine, which was finally protected as a tosyl (3a-b) or a mesyl group (3c-d). ${ }^{20-21}$ Our preliminary studies began by reacting cyclohexadiene 3a with phenyl iodide $4 \mathbf{a}$ in the presence of $\operatorname{Pd}(\mathrm{OAc})_{2}$ under cationic Heck reaction conditions. ${ }^{22}$ We observed no trace of heterocyclization product such as II in the mixture, which instead contained mainly starting material in $44 \%$ yield, an unexpected cyclopropane containing product 5a (31\%) and a third product (25\%), which structure could not be determined (Scheme 2). 5a was obtained as a single diastereomer with the tentative stereochemistry as shown.

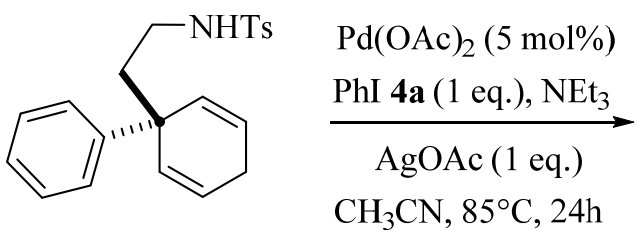

3a

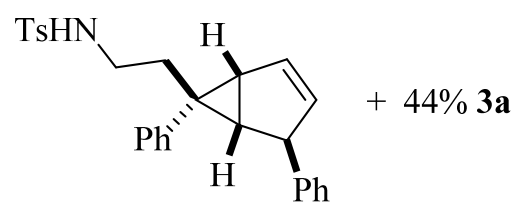

5a (31\%)

Scheme 2. Reaction of dienes 3a and PhI under Heck conditions.

5a was finally obtained with higher efficiency using $\mathrm{Ag}_{2} \mathrm{CO}_{3}$ instead of $\mathrm{AgOAc}$ and in presence of triphenylphosphine, albeit as a 9:1 mixture of isomers (Table 1, entry 1). The reaction was extended to other aryl iodides including $\mathbf{4 b - c}$ and arylcyclohexadienes $\mathbf{3 b}$-d. Under 
these conditions, cyclopropanes 5b-e were obtained as single isolated diastereomers with moderate to good yields, along with recovered starting material in some cases (entries 3-4).

Table 1. Reaction of dienes 3a-d and aryl iodides 4a-c under Heck conditions
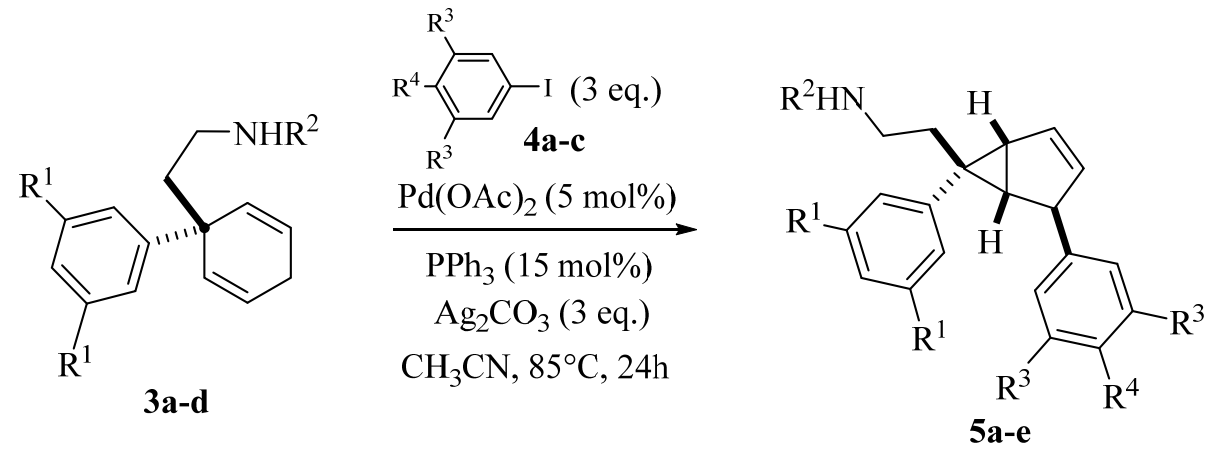

\begin{tabular}{ccccccccc}
\hline Entry & $\mathbf{3}$ & $\mathrm{R}_{1}$ & $\mathrm{R}_{2}$ & $\mathbf{4}$ & $\mathrm{R}_{3}$ & $\mathrm{R}_{4}$ & $\mathbf{5}$ & ${\text { Yield }(\%)^{\mathrm{a}}}^{\mathrm{a}}$ \\
\hline 1 & $\mathbf{3 a}$ & $\mathrm{H}$ & $\mathrm{Ts}$ & $\mathbf{4 a}$ & $\mathrm{H}$ & $\mathrm{H}$ & $\mathbf{5 a}$ & 77 \\
2 & $\mathbf{3 b}$ & $\mathrm{OMe}$ & $\mathrm{Ts}$ & $\mathbf{4 b}$ & $\mathrm{OMe}$ & $\mathrm{H}$ & $\mathbf{5 b}$ & 52 \\
3 & $\mathbf{3 c}$ & $\mathrm{H}$ & $\mathrm{Ms}$ & $\mathbf{4 b}$ & $\mathrm{OMe}$ & $\mathrm{H}$ & $\mathbf{5 c}$ & $39(51, \mathrm{brsm})^{\mathrm{b}}$ \\
4 & $\mathbf{3 d}$ & $\mathrm{OMe}$ & $\mathrm{Ms}$ & $\mathbf{4 b}$ & $\mathrm{OMe}$ & $\mathrm{H}$ & $\mathbf{5 d}$ & $38(49, \mathrm{brsm})^{\mathrm{b}}$ \\
5 & $\mathbf{3 a}$ & $\mathrm{H}$ & $\mathrm{Ts}$ & $\mathbf{4 c}$ & $\mathrm{H}$ & $\mathrm{OMe}$ & $\mathbf{5 e}$ & 37 \\
\hline
\end{tabular}

${ }^{\mathrm{a}}$ Isolated yields. ${ }^{\mathrm{b}}$ Yields based on recovered starting material.

Our efforts to obtain crystals of 5a-e suitable for X-ray structure determination unfortunately failed. ${ }^{13} \mathrm{C}$ NMR (DEPT) clearly shows the presence of two aliphatic $\mathrm{CH}_{2}$ and three aliphatic $\mathrm{CH}$, with chemical shifts in good agreement with a bicyclic structure bearing a cyclopropane ring. Complete 2D-NMR analysis of 5a showed an absence of NOESY correlation between the benzylic proton and the contiguous cyclopropane $\mathrm{CH}$, leading to the conclusion that these protons were trans to each other as in the structure shown. Further support to the above structure was obtained through DFT calculations of the ${ }^{13} \mathrm{C}$ NMR spectrum of 5c, using a standardized method (B3LYP/6-311+G(2d,p)-SCRF//B3LYP/6-311+G(2d,p)) and established scaling factors. ${ }^{23}$ The RMS deviation obtained for this compound (2.0338) was shown to be below the deviation obtained with the author's probe set used to validate the method (2.7992), thus supporting the above structure for 5c. Computed chemical shifts and method parameters have been added after the description of $5 c$ in the experimental section.

A tentative mechanism to rationalize the formation of cyclopropanes 5a-e is proposed in Figure 2 (Path A), which involves an oxidative addition of the catalyst into the C-I bond of the aryl iodides 4a-c, followed by coordination of the $\mathrm{Pd}(\mathrm{II})$ to the nitrogen side chain. The addition of the resulting aryl-palladium species Ia onto one of the $\mathrm{C}=\mathrm{C}$ bond of the diene 3a-d is then facilitated by this chelation thus controlling the regio- and stereoselectivity of the process. The syn-addition of the resulting aryl-palladium IIa generates an alkyl-palladium IIIa, which cannot 
evolve through a $\beta$-hydride elimination, but instead insert into the second olefin, forming the cyclopropane ring in IVa. ${ }^{24,25} \beta$-elimination finally affords 5a-e and an hydrido-palladium species, which returns the $\operatorname{Pd}(0)$ catalyst. Starting the mechanism with a coordination of the nitrogen on palladium seems reasonable as only compounds bearing a sulfonyl moiety on nitrogen react by this way. Cyclohexadienes bearing amides or carbamates substituents remained unchanged under the reaction conditions. Sulfonamides being known as palladium ligands, their presence would be a prerequisite for this unusual reactivity profile.



Figure 2. Putative mechanism for the formation of 5a-e and 6 from 1-arylcyclohexa-2,5-dienes 3a-d.

When the reaction was performed with electron-poor aryl iodides such as p-nitrophenyl iodide, no reaction took place. Interestingly, when cyclohexadiene 3a was reacted with 2iodothiophene 4d, no trace of the corresponding cyclopropane was formed, but instead the product of heterocyclization 6 was observed, along with unreacted starting material (Scheme 3). 6 was previously prepared from $3 \mathbf{a}^{10}$ using Wacker type conditions $\left(\mathrm{Pd}(\mathrm{OAc})_{2}\right.$, pyridine, $\mathrm{O}_{2}$ in xylene at $80^{\circ} \mathrm{C}$ ). The formation of 6 may proceed through heterocyclization prior to $\beta$-hydride elimination as shown in Figure 2 (Path B). It is noteworthy that no Heck reaction on the resulting diene was observed. Although the yield of $\mathbf{6}$ is moderate, it has not been optimized and clearly indicates that with our substrates, 2-iodothiophene could be a good candidate to perform efficiently the reoxidation of $\operatorname{Pd}(0)$ in an aza-Wacker type process, as illustrated in Scheme 1. 


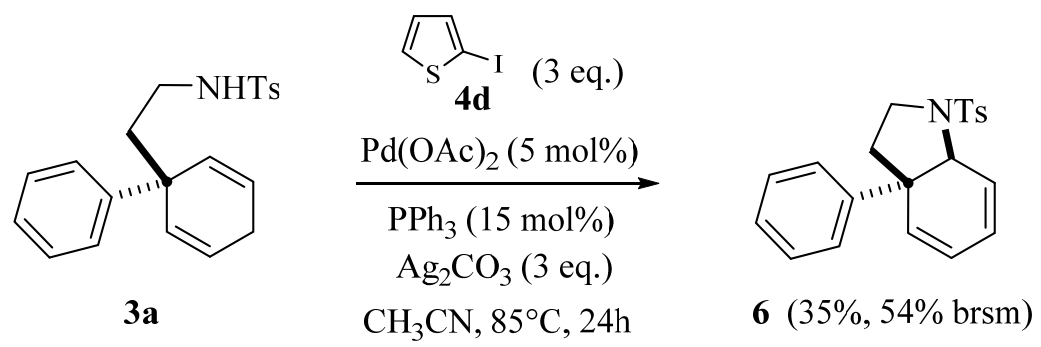

Scheme 3. Reaction of diene 3a and 2-iodothiophene 4c under Heck conditions.

\section{Conclusions}

We reported above a unique reactivity of 1,1-disubstituted cyclohexa-2,5-dienes in the presence of electron-rich aryl iodides under $\operatorname{Pd}(0)$ catalysis. Under these Heck reaction conditions, one observed the unexpected formation of bicyclo[3.1.0] systems in a diastereocontrolled manner in moderate to good yields. To our knowledge, such a process has no precedent in the literature. When 2-iodothiophene 4d was used as the aryl iodide partner, the cyclopropane was not formed, but instead we obtained an heterocycle (e.g. 6) resulting from an aza-Wacker type process, the aryl iodide likely serving as to reoxidize $\mathrm{Pd}(0)$ into $\mathrm{Pd}(\mathrm{II})$ through an oxidative addition.

\section{Experimental Section}

General. ${ }^{1} \mathrm{H}$ NMR and ${ }^{13} \mathrm{C}$ NMR were recorded on a Brüker Avance $300\left({ }^{1} \mathrm{H}: 300 \mathrm{MHz},{ }^{13} \mathrm{C}\right.$ : $75.5 \mathrm{MHz})$, Brüker AC-250 FT $\left({ }^{1} \mathrm{H}: 250 \mathrm{MHz},{ }^{13} \mathrm{C}: 62.9 \mathrm{MHz}\right)$, using solvent peak as internal reference or apparatus SR. The chemical shifts $(\delta)$ and coupling constants $(J)$ are expressed in ppm and hertz respectively. " $a$ " means "apparent" for close coupling constant and the number of equivalent group are indicated after the sign " $\mathrm{x}$ ". Carbon attribution $\mathrm{C}, \mathrm{CH}, \mathrm{CH}_{2}$ and $\mathrm{CH}_{3}$ were determined by ${ }^{13} \mathrm{C}$, DEPT 135 and HMQC experiments. InfraRed (IR) spectra were recorded on a Perkin-Elmer Paragon 1000 FT-IR spectrophotometer. Melting points were uncorrected and determined by using a Büchi-Totolli apparatus. Merck silica gel $(0.043-0.063 \mathrm{~mm})$ was used for flash chromatography. $\mathrm{CH}_{2} \mathrm{Cl}_{2}$ (DCM) and acetonitrile were distilled under $\mathrm{CaH}_{2}$. All reactions were carried out under nitrogen. Other reagents and starting materials were directly used as obtained commercially.

$N$-[2-(1-Phenyl-cyclohexa-2,5-dienyl)-ethyl]-methanesulfonamide (3c). To a solution of 2-(1Phenyl-cyclohexa-2,5-dienyl)-ethylamine ${ }^{20,21}(536 \mathrm{mg}, 2.69 \mathrm{mmol})$ in DCM (26 mL) was added triethylamine $(760 \mu \mathrm{L}, 5.42 \mathrm{mmol})$ and methanesulfonyl chloride $(210 \mu \mathrm{L}, 2.71 \mathrm{mmol})$ at $0^{\circ} \mathrm{C}$. After stirring overnight at room temperature, the organic layer was washed with water then brine, dried over sodium sulfate and filtered. Evaporation of the solvents gave a white solid which was 
purified through silica gel chromatography (Petroleum ether/EtOAc 80:20) to give $\mathrm{N}$-[2-(1phenyl-cyclohexa-2,5-dienyl)-ethyl]-methanesulfonamide 3c as a white solid (737 mg, 98\%). Mp $=94-96{ }^{\circ} \mathrm{C}$. IR (solid, KBr, $v_{\max } \mathrm{cm}^{-1}$ ): 3288, 1314, 1153, 1060, 728, 703, 595, 525, 514. ${ }^{1} \mathrm{H}$ NMR (300 MHz, $\left.\mathrm{CDCl}_{3}\right): \delta_{\mathrm{H}} 2.10-2.15\left(2 \mathrm{H}, \mathrm{m}, \mathrm{CH}_{2} \mathrm{CH}_{2} \mathrm{~N}\right), 2.68-2.74\left(2 \mathrm{H}, \mathrm{m}\right.$, bis allylic $\left.\mathrm{CH}_{2}\right)$, $2.94\left(3 \mathrm{H}, \mathrm{s}, \mathrm{NSO}_{2} \mathrm{CH}_{3}\right), 3.15-3.22\left(2 \mathrm{H}, \mathrm{m}, \mathrm{CH}_{2} \mathrm{~N}\right), 4.83(1 \mathrm{H}$, broad s, $\mathrm{NH})$, 5.61-5.66 $(2 \mathrm{H}, \mathrm{m}$, $\mathrm{CH}$ x 2 olefinic), $5.92\left(2 \mathrm{H}, \mathrm{dta},{ }^{3} J_{H H} 10.5,{ }^{4} J_{H H} 3.4\right.$ and $3.4 \mathrm{~Hz}, \mathrm{CH}$ x 2 olefinic), 7.17-7.23 (1H, $\mathrm{m}, \mathrm{CH}$ aromatic), 7.29-7.36 (4H, m, $4 \mathrm{CH}$ aromatic). ${ }^{13} \mathrm{C} \mathrm{NMR}\left(\mathrm{CDCl}_{3}, 75.5 \mathrm{MHz}\right): \delta_{\mathrm{C}} 26.0\left(\mathrm{CH}_{2}\right.$ bis-allylic), $39.8\left(\underline{\mathrm{CH}}_{2} \mathrm{CH}_{2} \mathrm{~N}\right), 40.1\left(\mathrm{NSO}_{2} \mathrm{CH}_{3}\right), 40.8\left(\mathrm{CH}_{2} \mathrm{~N}\right), 43.0$ (C aliphatic), $124.7(\mathrm{CH} \times 2$, olefinic), 126.4 (CH aromatic), 126.4, 128.5 (2CH x 2 aromatic), 131.7 ( $\mathrm{CH}$ x 2 olefinic), 147.1 (C aromatic). Anal. calcd for $\mathrm{C}_{15} \mathrm{H}_{19} \mathrm{NO}_{2} \mathrm{~S}$ (277.11): C, 64.95; H, 6.90; N, 5.05; S, 11.56. Found: C, 65.17; H, 7.18; N, 4.88; S, 11.79.

\section{$N$-\{2-[1-(3,5-Dimethoxy-phenyl)-cyclohexa-2,5-dienyl]-ethyl\}-methanesulfonamide (3d).}

To a solution of 2-[1-(3,5-dimethoxyphenyl)-cyclohexa-2,5-dienyl]-ethylamine ${ }^{20,21}$ (298 mg, $1.15 \mathrm{mmol})$ in DCM $(11 \mathrm{~mL})$ were added triethylamine $(480 \mu \mathrm{L}, 3.45 \mathrm{mmol})$ and methanesulfonyl chloride ( $90 \mu \mathrm{L}, 2.30 \mathrm{mmol})$. After stirring for 2 hours, the organic layer was washed with water, dried over sodium sulfate, filtrated, and the solvents were evaporated. The crude mixture was purified through silica gel chromatography (Petroleum ether/EtOAc 65:35) to give 3d as a colorless viscous oil (328 mg, 85\%). IR (film, $\left.\mathrm{NaCl}, v_{\max } \mathrm{cm}^{-1}\right)$ : 3307, 2937, 1614 , $1455,1316,1203,1149,1062,975,839,797 .{ }^{1} \mathrm{H}$ NMR $\left(300 \mathrm{MHz}, \mathrm{CDCl}_{3}\right): \delta_{\mathrm{H}} 2.06\left(2 \mathrm{H}, \mathrm{t},{ }^{3} J_{\mathrm{HH}}\right.$ $\left.5.3 \mathrm{~Hz}, \mathrm{CH}_{2} \mathrm{CH}_{2} \mathrm{~N}\right), 2.69$ (2H, broad s, $\mathrm{CH}_{2}$ bis-allylic), $2.92\left(3 \mathrm{H}, \mathrm{s}, \mathrm{NSO}_{2} \mathrm{CH}_{3}\right), 3.11-3.19(2 \mathrm{H}$, $\left.\mathrm{m}, \mathrm{CH}_{2} \mathrm{~N}\right), 3.76\left(6 \mathrm{H}, \mathrm{s}, \mathrm{OCH}_{3} \times 2\right), 4.64(1 \mathrm{H}$, broad s, $\mathrm{NH}), 5.59-5.63(2 \mathrm{H}, \mathrm{m}, \mathrm{CH}$ x 2 olefinic), 5.87-5.93 $(2 \mathrm{H}, \mathrm{m}, \mathrm{CH} \times 2$ olefinic), $6.30(1 \mathrm{H}, \mathrm{s}, \mathrm{CH}$ aromatic), $6.48(2 \mathrm{H}, \mathrm{s}, \mathrm{CH} \times 2$ aromatic). ${ }^{13} \mathrm{C}$ NMR (75.5 MHz, CDCl 3$): \delta_{\mathrm{C}} 26.0\left(\mathrm{CH}_{2}\right.$ bis-allylic), $40.1\left(\mathrm{CH}_{2} \mathrm{CH}_{2} \mathrm{~N}\right), 40.3\left(\mathrm{NSO}_{2} \mathrm{CH}_{3}\right)$, $40.7\left(\mathrm{CH}_{2} \mathrm{~N}\right), 43.2$ (C aliphatic), $55.4\left(\mathrm{OCH}_{3} \times 2\right), 97.8(\mathrm{CH}$ aromatic), $105.1(\mathrm{CH} \times 2$, aromatic), 124.9 ( $\mathrm{CH} \times 2$, olefinic), 131.5 ( $\mathrm{CH} \times 2$, olefinic), 149.7 (C aromatic), 160.9 (C x 2, aromatic). MS(ESI) $m / z$ (\%): 360 (M+Na, 100). HRMS calcd for $(\mathrm{M}+\mathrm{Na}) \mathrm{C}_{17} \mathrm{H}_{23} \mathrm{NNaO}_{4} \mathrm{~S}: 360.1245$; found $360.1232(3.6 \mathrm{ppm})$.

\section{$N$-[2-(4,6-Diphenyl-bicyclo[3.1.0]hex-2-en-6-yl)-ethyl]-4-methyl-benzenesulfonamide (5a).}

In a dry two necked flask were introduced $3 \mathbf{a}(211 \mathrm{mg}, 0.60 \mathrm{mmol})$, silver carbonate $(247 \mathrm{mg}$, $0.90 \mathrm{mmol})$, triphenylphosphine $(23 \mathrm{mg}, 0.09 \mathrm{mmol})$, palladium acetate $(7 \mathrm{mg}, 0.03 \mathrm{mmol})$ and acetonitrile $(30 \mathrm{~mL})$. Phenyl iodide $4 \mathbf{a}(100 \mu \mathrm{L}, 0.9 \mathrm{mmol})$ was added and the mixture was warmed to $85^{\circ} \mathrm{C}$ and stirred overnight. The mixture was filtered through celite, which was then washed with ethyl acetate. Evaporation of the solvents led to a paste, which was purified through silica gel chromatography (Petroleum ether/EtOAc 85:15), affording 5a as a viscous oil (198 $\mathrm{mg}$, 77\%). IR (film, $\mathrm{KBr}, v_{\max }, \mathrm{cm}^{-1}$ ): 3286, 3024, 2923, 1599, 1494, 1444, 1325, 1160, 1094, 703, 664. ${ }^{1} \mathrm{H}$ NMR $\left(300 \mathrm{MHz}, \mathrm{CDCl}_{3}\right): \delta_{\mathrm{H}} 1.41-1.51\left(1 \mathrm{H}, \mathrm{m}, \mathrm{C} \underline{\mathrm{H}} a \mathrm{H} x \mathrm{CH}_{2} \mathrm{~N}\right), 1.78-1.88(2 \mathrm{H}, \mathrm{m}$, $\mathrm{CH} a \underline{\mathrm{H}} x \mathrm{CH}_{2} \mathrm{~N}$ and $\mathrm{CH}$ cyclopropane), $2.29\left(1 \mathrm{H}, \mathrm{dt} a, J_{\mathrm{HH}} 6.0,2.6\right.$ and $2.6 \mathrm{~Hz}, \mathrm{CH}$ cyclopropane), $2.43\left(3 \mathrm{H}, \mathrm{s}, \mathrm{CH}_{3}\right), 2.94-2.87\left(2 \mathrm{H}, \mathrm{m}, \mathrm{CH}_{2} \mathrm{~N}\right), 3.23(1 \mathrm{H}$, broad s, $\mathrm{CH}$ cyclopropane $), 4.59(1 \mathrm{H}, \mathrm{t}$, 
$\left.{ }^{3} J_{\mathrm{HH}} 6.0 \mathrm{~Hz}, \mathrm{NH}\right), 5.15\left(1 \mathrm{H}, \mathrm{ddd}, J_{\mathrm{HH}} 5.3,2.2\right.$ and $0.8 \mathrm{~Hz}$, olefinic $\left.\mathrm{H}\right), 5.87\left(1 \mathrm{H}, \mathrm{dta}, J_{\mathrm{HH}} 5.3,2.3\right.$ and $2.3 \mathrm{~Hz}$, olefinic $\mathrm{H}), 7.07-7.33\left(12 \mathrm{H}, 10 \mathrm{CH}\right.$ aromatic $+2 \mathrm{CH}$ tosyl), $7.65\left(2 \mathrm{H}, \mathrm{d},{ }^{3} J_{\mathrm{HH}} 8.3 \mathrm{~Hz}\right.$, $2 \mathrm{CH}$ tosyl). ${ }^{13} \mathrm{C} \mathrm{NMR}\left(75.5 \mathrm{MHz}, \mathrm{CDCl}_{3}\right): \delta_{\mathrm{C}} 21.6\left(\mathrm{CH}_{3}\right.$ tosyl), $37.0(\mathrm{CH}$ cyclopropane), 37.5 (C aliphatic), 38.0 ( $\mathrm{CH}$ cyclopropane), 41.1, $41.2\left(2 \mathrm{CH}_{2}, \mathrm{CH}_{2} \mathrm{CH}_{2} \mathrm{~N}\right), 51.1(\mathrm{CH}$ allylic), 126.5, 126.6 (2CH, phenyl), 127.1, 127.9, 128.1, 128.6, 129.7 (5CH x 2 aromatic), 131.4 (CH olefinic), 131.7 (CH x 2, aromatic), 134.3 (CH, olefinic), 137.0, 137.5, 143.4, 144.2 (4C aromatic). MS(SIMS) m/z (\%): 452 (M+Na, 100), 429 (M), 274 (33), 259 (47), 245 (77). HRMS calcd for (M) $\mathrm{C}_{27} \mathrm{H}_{27} \mathrm{NO}_{2} \mathrm{~S}$ : 429.1763; found 429.1758 (1.2 ppm).

\section{$N$-\{2-[4,6-Bis-(3,5-dimethoxyphenyl)-bicyclo[3.1.0]hex-2-en-6-yl]-ethyl\}-4-methylbenzene-} sulfonamide (5b). In a dry two necked flask were introduced $N$ - $\{2-[1-(3,5$-dimethoxyphenyl)cyclohexa-2,5-dienyl]-ethyl\}-4-methyl-benzenesulfonamide $3 \mathbf{b}$ (66 mg, $0.168 \mathrm{mmol}$ ), silver carbonate (93 mg, $0.336 \mathrm{mmol}$ ), triphenylphosphine (6.6 $\mathrm{mg}, 0.025 \mathrm{mmol}), 3,5$-dimethoxyiodobenzene $4 \mathbf{b}(89 \mathrm{mg}, 0.336)$, palladium acetate $(1.9 \mathrm{mg}, 0.008 \mathrm{mmol})$ and acetonitrile $(8.5$ $\mathrm{mL}$ ). The mixture was warmed to $85{ }^{\circ} \mathrm{C}$ and stirred for 20 hours. The mixture was filtered through celite, which was then washed with ethyl acetate. Evaporation of the solvents led to a paste, which was purified through silica gel chromatography (Petroleum ether/EtOAc 80:20), affording $\mathbf{5 b}$ as a viscous oil (48 mg, 52\%). IR (film, KBr, $\left.v_{\max } \mathrm{cm}^{-1}\right)$ : 2937, 1594, 1458, 1425 , 1325, 1204, 1155, 1064, 839, 732. ${ }^{1} \mathrm{H}$ NMR (300 MHz, $\left.\mathrm{CDCl}_{3}\right): \delta_{\mathrm{H}} 1.38-1.50(1 \mathrm{H}, \mathrm{m}$, C$\left.a \mathrm{H} x \mathrm{CH}_{2} \mathrm{~N}\right), 1.72-1.84\left(2 \mathrm{H}, \mathrm{m}, \mathrm{CH} a \underline{\mathrm{H}} x \mathrm{CH}_{2} \mathrm{~N}\right.$ and $\mathrm{CH}$ cyclopropane), 2.21-2.26 (1H, m, $\mathrm{CH}$ cyclopropane), $2.41\left(3 \mathrm{H}, \mathrm{s}, \mathrm{CH}_{3}\right.$ tosyl), $2.91\left(2 \mathrm{H}, \mathrm{qa},{ }^{3} \mathrm{~J}_{\mathrm{HH}} 7.3 \mathrm{~Hz}, \mathrm{CH}_{2} \mathrm{~N}\right), 3.28(1 \mathrm{H}$, broad s, $\mathrm{CH}$ cyclopropane), $3.77\left(12 \mathrm{H}, 2 \mathrm{OCH}_{3} \mathrm{x} 2\right), 4.46(1 \mathrm{H}$, broad s, $\mathrm{NH}), 5.20\left(1 \mathrm{H}, \mathrm{d},{ }^{3} J_{\mathrm{HH}} 5.3 \mathrm{~Hz}, \mathrm{CH}\right.$ olefinic), 5.82-5.84 (1H, m, CH olefinic), $6.22(2 \mathrm{H}, \mathrm{s}, 2 \mathrm{CH}$ aromatic) $6.32(4 \mathrm{H}, \mathrm{s}, 2 \mathrm{CH} \times 2$ aromatic), $7.25\left(2 \mathrm{H}, \mathrm{d},{ }^{3} J_{\mathrm{HH}} 8.3 \mathrm{~Hz}, \mathrm{CH} \times 2\right.$ tosyl), $7.63\left(2 \mathrm{H}, \mathrm{d},{ }^{3} J_{\mathrm{HH}} 8.3 \mathrm{~Hz}, \mathrm{CH} \times 2\right.$ tosyl). ${ }^{13} \mathrm{C}$ NMR (75.5 MHz, $\left.\mathrm{CDCl}_{3}\right): \delta_{\mathrm{C}} 21.6\left(\mathrm{CH}_{3}\right.$ tosyl), 37.1, 38.0 (2CH cyclopropane), 38.1 (C aliphatic), 41.2, $41.3\left(2 \mathrm{CH}_{2}, \mathrm{CH}_{2} \mathrm{CH}_{2} \mathrm{~N}\right), 51.2\left(\mathrm{CH}\right.$ allylic), 55.4, $55.5\left(2 \mathrm{OCH}_{3} \times 2\right), 98.2,98.5$ (2CH aromatic), 105.9, 110.0 (2CH x 2 aromatic), 127.1, 129.8 (2CH x 2 tosyl), 131.6, 134.2 (2CH olefinic), 137.0, 140.0, 143.4, 146.7 (4C aromatic), 160.5, 161.0 (2C x 2 aromatic). MS(ESI) m/z (\%): $1121(2 \mathrm{M}+\mathrm{Na}, 22), 588(\mathrm{M}+\mathrm{K}, 13), 572(\mathrm{M}+\mathrm{Na}, 100)$. HRMS calcd for $[\mathrm{M}+\mathrm{Na}]^{+} \mathrm{C}_{31} \mathrm{H}_{35} \mathrm{NNaO}_{6} \mathrm{~S}$ : 572.2083; found 572.2082 (0 ppm).

\section{$N$-\{2-[4-(3,5-Dimethoxy-phenyl)-6-phenyl-bicyclo[3.1.0]hex-2-en-6-yl]-ethyl\}-methane-}

sulfonamide (5c). In a dry two necked flask were introduced $N$-[2-(1-Phenyl-cyclohexa-2,5dienyl)-ethyl]-methanesulfonamide 3c (169 mg, $0.610 \mathrm{mmol})$, silver carbonate (336 mg, 1.22 mmol), triphenylphosphine (24 mg, $0.092 \mathrm{mmol})$, 3,5-dimethoxy-iodobenzene $4 \mathbf{b}$ (322 $\mathrm{mg}, 1.22$ $\mathrm{mmol})$, palladium acetate $(6.8 \mathrm{mg}, 0.030 \mathrm{mmol})$ and acetonitrile $(24.5 \mathrm{~mL})$. The mixture was warmed to $85^{\circ} \mathrm{C}$ and stirred for 16 hours. The mixture was filtered through celite, which was then washed with ethyl acetate. Evaporation of the solvents led to a paste, which was purified through silica gel chromatography (Petroleum ether/EtOAc 70:30), affording 5c as a white solid (98 mg, 39\%). $\mathrm{Mp}=123-125{ }^{\circ} \mathrm{C}$. IR (solid, $\mathrm{KBr}, v_{\max } \mathrm{cm}^{-1}$ ): 3295, 2936, 2255, 1606, 1454, 
1316, 1203, 1149, 1064, 973, 910, 777, 735, 710. ${ }^{1} \mathrm{H}$ NMR $\left(300 \mathrm{MHz}, \mathrm{CDCl}_{3}\right): \delta_{\mathrm{H}} 1.53-1.63$ $\left(1 \mathrm{H}, \mathrm{m}, \mathrm{CH} a \mathrm{H} x \mathrm{CH}_{2} \mathrm{~N}\right), 1.90\left(1 \mathrm{H}, \mathrm{d},{ }^{3} \mathrm{~J}_{\mathrm{HH}} 6.0 \mathrm{~Hz}, \mathrm{CH}\right.$ cyclopropane), 1.93-2.00 (1H, m, $\left.\mathrm{CHa} \underline{\mathrm{H}} x \mathrm{CH}_{2} \mathrm{~N}\right), 2.36\left(1 \mathrm{H}, \mathrm{dta}, J_{\mathrm{HH}} 6.0,2.5\right.$ and $2.5 \mathrm{~Hz}, \mathrm{CH}$ cyclopropane), 2.82 (3H, s, $\left.\mathrm{NSO}_{2} \mathrm{CH}_{3}\right), 3.00-3.10\left(2 \mathrm{H}, \mathrm{m}, \mathrm{CH}_{2} \mathrm{~N}\right), 3.19-3.23(1 \mathrm{H}, \mathrm{m}, \mathrm{CH}$ cyclopropane $), 3.78\left(6 \mathrm{H}, \mathrm{s}, \mathrm{OCH}_{3} \mathrm{x}\right.$ 2), $4.43\left(1 \mathrm{H}\right.$, broad s, NH), $5.16\left(1 \mathrm{H}\right.$, ddd, $J_{\mathrm{HH}} 5.3,2.2$ and $1.5 \mathrm{~Hz}, \mathrm{CH}$ olefinic), 5.91 (1H, dta, $J$ $=5.3,2.0$ and $2.0 \mathrm{~Hz}, \mathrm{CH}$ olefinic), $6.33\left(1 \mathrm{H}, \mathrm{t},{ }^{4} J_{\mathrm{HH}} 2.3 \mathrm{~Hz}, \mathrm{CH}\right.$ aromatic $), 6.36\left(1 \mathrm{H}, \mathrm{t},{ }^{4} J_{\mathrm{HH}} 2.3\right.$ $\mathrm{Hz}, \mathrm{CH} \times 2$ aromatic), 7.17-7.35 (5H, m, 5CH aromatic). $\left.{ }^{13} \mathrm{C} \mathrm{NMR} \mathrm{(75.5} \mathrm{MHz}, \mathrm{CDCl}_{3}\right): 36.9$ (CH cyclopropane), 37.7 (C aliphatic), 37.9 ( $\mathrm{CH}$ cyclopropane), $40.0\left(\mathrm{NSO}_{2} \mathrm{CH}_{3}\right),\left(\mathrm{CH}_{2} \mathrm{~N}\right), 41.7$ $\left(\mathrm{CH}_{2} \mathrm{CH}_{2} \mathrm{~N}\right), 51.3\left(\mathrm{CH}\right.$ allylic), $55.4\left(\mathrm{OCH}_{3} \times 2\right), 98.5(\mathrm{CH}$ aromatic), $105.8(\mathrm{CH} \times 2$ aromatic), 126.6 ( $\mathrm{CH}$ aromatic), 128.2 ( $\mathrm{CH} \times 2$ aromatic), 131.6 ( $\mathrm{CH}$ olefinic), 131.7 ( $\mathrm{CH} \times 2$ aromatic), 134.1 (CH olefinic), 137.5, 146.6 (2C aromatic), 160.9 (C x 2 aromatic). MS(SIMS) m/z (\%): $436(\mathrm{M}+\mathrm{Na}, 35), 414(\mathrm{M}+\mathrm{H}, 100), 305$ (35). HRMS calcd for $(\mathrm{M}+\mathrm{H}) \mathrm{C}_{23} \mathrm{H}_{28} \mathrm{NO}_{4} \mathrm{~S} 413.1661$; found 413.1655 (1.5 ppm).

\section{DFT computed ${ }^{13} \mathrm{C}$ NMR spectrum of $5 \mathrm{c}$}

Gaussian09 was used with B3LYP/6-311+G(2d,p)-SCRF//B3LYP/6-311+G(2d,p) basis set. The solvent (chloroform) effect has been accounted with $\mathrm{SCRF}=(\mathrm{smd}$,solvent=chloroform $)$.

The following equation is used to produce scaled chemical shifts $(\delta)$ from computed isotropic shielding constants $(\sigma)$. Scaling factors from ref. 23 were used.

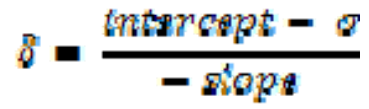

With slope $=-1.0451$ and intercept $=182.3835\left(\mathrm{R}^{2}=0.9986\right)$.

Computed $\delta_{\mathrm{C}}\left(\right.$ experimental $\left.\delta_{\mathrm{C}}\right) 36.9$ (36.9), 37.1 (37.7), 39.8 (41.2), 40.0 (37.9), 42.1 (41.7), 44.3 (40.0), 54.3 (51.3), 52.9 (55.4), 95.0 (98.5), 102.5 (105.8), 125.0 (126.6), 126.9 (128.2), 131.0 (131.6), 133.8 (131.7), 134.5 (134.1), 138.5 (137.5), 147.2 (146.6), 161.4 (160.9).

\section{$N$-\{2-[4,6-Bis-(3,5-dimethoxy-phenyl)-bicyclo[3.1.0]hex-2-en-6-yl]-ethyl\}-}

methanesulfonamide (5d). In a dry two necked flask equipped with a condenser were introduced iodo-3,5-dimethoxybenzene $4 \mathbf{b}(517 \mathrm{mg}, 1.96 \mathrm{mmol})$, silver carbonate $(538 \mathrm{mg}, 1.96$ mmol), palladium acetate $(7.3 \mathrm{mg}, 0.033 \mathrm{mmol})$, triphenylphosphine $(26 \mathrm{mg}, 0.098 \mathrm{mmol})$ then a solution of (N-\{2-[1-(3,5-dimethoxy-phenyl)-cyclohexa-2,5-dienyl]-ethyl\}-methanesulfonamide 3d $(220 \mathrm{mg}, 0.653 \mathrm{mmol})$ in acetonitrile $(26 \mathrm{~mL})$. The mixture was warmed to $85{ }^{\circ} \mathrm{C}$ for 14 hours. The mixture was filtered through celite, which was then washed with ethyl acetate. Evaporation of the solvents led to a paste, which was purified through silica gel chromatography (25 g, Petroleum ether/EtOAc 70:30), affording, first, starting material 3d (48 mg, 22\%) then 5d as a viscous oil $(\Delta \mathrm{R} f \leq 0.1)(117 \mathrm{mg}, 38 \%)$. IR (film, $\left.\mathrm{NaCl}, v_{\max }, \mathrm{cm}^{-1}\right): 3298 \mathrm{~cm}^{-1}, 2937,1594$, 1456, 1320, 1204, 1154, 1064, 839, 777, 735. ${ }^{1} \mathrm{H}$ NMR (300 MHz, $\left.\mathrm{CDCl}_{3}\right): \delta_{H} 1.53-1.62(1 \mathrm{H}, \mathrm{m}$, C$\left.a \mathrm{H} x \mathrm{CH}_{2} \mathrm{~N}\right), 1.85-1.98\left(2 \mathrm{H}, \mathrm{m}, \mathrm{CH} a \underline{\mathrm{H}} x \mathrm{CH}_{2} \mathrm{~N}\right.$ and $\mathrm{CH}$ cyclopropane), 2.31-2.35 (1H, m, $\mathrm{CH}$ cyclopropane), $2.85\left(3 \mathrm{H}, \mathrm{s}, \mathrm{NSO}_{2} \mathrm{CH}_{3}\right), 3.05-3.11\left(2 \mathrm{H}, \mathrm{qa},{ }^{3} \mathrm{~J}_{\mathrm{HH}} 6.6 \mathrm{~Hz}, \mathrm{CH}_{2} \mathrm{~N}\right), 3.34(1 \mathrm{H}$, broad 
s, CH cyclopropane), $3.78\left(6 \mathrm{H}, \mathrm{s}, \mathrm{OCH}_{3} \times 2\right), 3.79\left(6 \mathrm{H}, \mathrm{s}, \mathrm{OCH}_{3} \times 2\right), 4.33(1 \mathrm{H}$, broad s, NH), 5.22-5.25 (1H, m, CH olefinic), 5.86-5.89 (1H, m, $\mathrm{CH}$ olefinic), 6.31-6.35 (6H, $\mathrm{m}, \mathrm{CH}$ aromatic). ${ }^{13} \mathrm{C}$ NMR (75.5 MHz, $\mathrm{CDCl}_{3}$ ): $\delta_{\mathrm{C}} 37.2$ (CH cyclopropane), 38.0 (C aliphatic), 38.1 (CH cyclopropane), $40.2\left(\mathrm{NSO}_{2} \mathrm{CH}_{3}\right), 41.4\left(\mathrm{CH}_{2} \mathrm{~N}\right), 41.7\left(\underline{\mathrm{CH}}_{2} \mathrm{CH}_{2} \mathrm{~N}\right), 51.2(\mathrm{CH}$ allylic $), 55.4$ $\left(2 \mathrm{OCH}_{3} \times 2\right), 98.1,98.5$ (2CH aromatic), 105.9, 110.1 (2CH x 2 aromatic), 131.5, $134.3(2 \mathrm{CH}$ olefinic), 140.0, 146.7 (2C aromatic), 160.6, 161.0 (2C x 2 aromatic). MS(ESI) m/z (\%): 496 (M+Na, 100). HRMS calcd for $(\mathrm{M}+\mathrm{Na}) \mathrm{C}_{25} \mathrm{H}_{31} \mathrm{NNaO}_{6} \mathrm{~S}$ 496.1770; found 496.1786 (-3.3 ppm).

\section{$N$-\{2-[4-(4-Methoxy-phenyl)-6-phenyl-bicyclo[3.1.0]hex-2-en-6-yl]-ethyl\}-4-methyl-} benzenesulfonamide (5e). In a dry two-necked flask were introduced 4-methyl- $N$-[2-(1-phenylcyclohexa-2,5-dienyl)-ethyl]-benzenesulfonamide 3a (71 $\mathrm{mg}, 0.200 \mathrm{mmol})$, silver carbonate (55 $\mathrm{mg}, 0.20 \mathrm{mmol})$, triphenylphosphine $(10 \mathrm{mg}, 0.04 \mathrm{mmol})$, palladium acetate $(2.3 \mathrm{mg}, 0.01 \mathrm{mmol})$ in acetonitrile $(8 \mathrm{~mL})$. 4-Methoxyphenyl iodide 4c $(47 \mathrm{mg}, 0.20 \mathrm{mmol})$ was added and the mixture was warmed to $85{ }^{\circ} \mathrm{C}$ and stirred overnight. The mixture was filtered through celite, which was then washed with ethyl acetate. Evaporation of the solvents led to a paste, which was purified through silica gel chromatography (Petroleum ether/EtOAc 90:10), affording 5e as a viscous oil (34 mg, 37\%). ${ }^{1} \mathrm{H}$ NMR $\left(250 \mathrm{MHz}, \mathrm{CDCl}_{3}\right): \delta_{\mathrm{H}} 1.38-1.50\left(1 \mathrm{H}, \mathrm{m}, \mathrm{CH} a \mathrm{H} x \mathrm{CH}_{2} \mathrm{~N}\right)$, 1.72-1.87 (2H, m, $\mathrm{CH} a \underline{\mathrm{H}} x \mathrm{CH}_{2} \mathrm{~N}$ and $\mathrm{CH}$ cyclopropane), $2.26\left(1 \mathrm{H}, \mathrm{dta}, J_{\mathrm{HH}} 6.1,2.4\right.$ and $2.4 \mathrm{~Hz}$, $\mathrm{CH}$ cyclopropane), $2.42\left(3 \mathrm{H}, \mathrm{s}, \mathrm{CH}_{3}\right.$ tosyl), $2.89\left(2 \mathrm{H}, \mathrm{qa},{ }^{3} \mathrm{~J}_{\mathrm{HH}} 7.6 \mathrm{~Hz}, \mathrm{CH}_{2} \mathrm{~N}\right), 3.17(1 \mathrm{H}$, broad s, CH cyclopropane), $3.79\left(3 \mathrm{H}, \mathrm{s}, \mathrm{OCH}_{3}\right), 4.45\left(1 \mathrm{H}, \mathrm{t},{ }^{3} \mathrm{~J}_{\mathrm{HH}} 6.1 \mathrm{~Hz}, \mathrm{NH}\right), 5.12\left(1 \mathrm{H}, \mathrm{ddd}, J_{\mathrm{HH}} 5.5\right.$, 2.1 and $1.2 \mathrm{~Hz}, \mathrm{CH}$ olefinic), $5.83\left(1 \mathrm{H}, \mathrm{dta}, J_{\mathrm{HH}} 5.5,2.1\right.$ and $2.1 \mathrm{~Hz}, \mathrm{CH}$ olefinic), $6.83(2 \mathrm{H}, \mathrm{d}$, ${ }^{3} J_{\mathrm{HH}} 8.6 \mathrm{~Hz}, \mathrm{CH} \times 2$ aromatic), 7.04-7.09 (4H, m, CH x 2 aromatic and $2 \mathrm{CH}$ tosyl), 7.21-7.29 (5H, m, $\mathrm{CH}$ aromatic), $7.63\left(2 \mathrm{H},{ }^{3} J_{\mathrm{HH}} 8.3 \mathrm{~Hz}, 2 \mathrm{CH}\right.$ tosyl). ${ }^{13} \mathrm{C} \mathrm{NMR}\left(62.9 \mathrm{MHz}, \mathrm{CDCl}_{3}\right): \delta_{\mathrm{C}}$ 21.6 ( $\mathrm{CH}_{3}$ tosyl), 36.8 ( $\mathrm{CH}$ cyclopropane), 37.7 (C aliphatic), 38.2 ( $\mathrm{CH}$ cyclopropane), 41.1, 41.2 $\left(2 \mathrm{CH}_{2}, \mathrm{CH}_{2} \mathrm{CH}_{2} \mathrm{~N}\right), 50.2\left(\mathrm{CH}\right.$ allylic), $55.4\left(\mathrm{OCH}_{3}\right), 113.9(\mathrm{CH} \times 2$ aromatic), $126.5(\mathrm{CH}$ aromatic), 127.1, 128.1, 128.8, 129.8 (4CH x 2 aromatic), 131.0 (CH olefinic), 131.7 (CH x 2 aromatic), 134.5 (CH olefinic), 136.3, 136.9, 137.6, 143.4, 158.3 (5C aromatic).

\section{Acknowledgements}

This work was supported by the MNERT through a PhD grant to RL. We also thank the CNRS for its help.

\section{References}

1. Abd Rahman, N.; Landais, Y. Curr. Org. Chem. 2002, 6, 1369 and references therein. http://dx.doi.org/10.2174/1385272023373347 
2. Studer, A.; Schleth, F. Synlett 2005, 3033 and references therein. http://dx.doi.org/10.1055/s-2005-921899

3. Nakahara, K.; Fujioka, H. Symmetry 2010, 2, 437 and references therein. http://dx.doi.org/10.3390/sym2020437

4. Walton, J. C.; Studer, A. Acc. Chem. Res. 2005, 38, 794 and references therein. http://dx.doi.org/10.1021/ar050089j

PMid:16231875

5. Lebeuf, R.; Robert, F.; Schenk, K.; Landais, Y. Org. Lett. 2006, 8, 4755. http://dx.doi.org/10.1021/ol0618353 PMid:17020295

6. Rousseau, G.; Robert, F.; Schenk, K.; Landais, Y. Org. Lett. 2008, 10, 4441. http://dx.doi.org/10.1021/ol8016885

PMid:18783229

7. Zeni, G.; Larock, R. C. Chem. Rev. 2004, 104, 2285.

http://dx.doi.org/10.1021/cr020085h

PMid:15137792

8. Stahl, S. S. Angew. Chem., Int. Ed. 2004, 43, 3400.

http://dx.doi.org/10.1002/anie.200300630

PMid:15221827

9. McDonald, R. I.; White, P. B.; Weinstein, A. B.; Tam, C. P.; Stahl, S. S. Org. Lett. 2011, 13, 2830 .

http://dx.doi.org/10.1021/ol200784y

PMid:21534607 PMCid:PMC3103601

10. Beniazza, R.; Dunet, J.; Robert, F.; Schenk, K.; Landais, Y. Org. Lett. 2007, 9, 3913. http://dx.doi.org/10.1021/o1701493k PMid:17824704

11. Guram, A. S.; Bei, X.; Turner, H. W. Org. Lett. 2003, 5, 2485.

http://dx.doi.org/10.1021/o10347287

PMid:12841761

12. Bouquillon, S.; Hénin, F.; Muzart, J. Organometallics 2000, 19, 1434.

http://dx.doi.org/10.1021/om990961q

13. Wolfe, J. P.; Rossi, M. A. J. Am. Chem. Soc. 2004, 126, 1620.

http://dx.doi.org/10.1021/ja0394838

PMid:14871078

14. Hay, M. B.; Wolfe, J. P. Tetrahedron Lett. 2006, 47, 2793.

http://dx.doi.org/10.1016/j.tetlet.2006.02.066

PMid:19789723 PMCid:PMC2752900

15. Ney, J. E.; Wolfe, J. P. Angew. Chem. Int. Ed. 2004, 43, 3605.

http://dx.doi.org/10.1002/anie.200460060

PMid:15293259 
16. Bertrand, M. B.; Wolfe, J. P. Tetrahedron 2005, 61, 6447.

http://dx.doi.org/10.1016/j.tet.2005.03.110

17. Bertrand, M. B.; Neukom, J. D.; Wolfe, J. P. J. Org. Chem. 2008, 73, 8851.

http://dx.doi.org/10.1021/jo801631v

PMid:18942792 PMCid:PMC2633938

18. Hay, M. B.; Wolfe, J. P. J. Am. Chem. Soc. 2005, 127, 16468.

http://dx.doi.org/10.1021/ja054754v

PMid:16305233 PMCid:PMC2750864

19. Schultz, D. M.; Wolfe, J. P. Synthesis 2012, 44, 351.

http://dx.doi.org/10.1055/s-0031-1289668

PMid:23243321 PMCid:PMC3521566

20. Lebeuf, R.; Dunet, J.; Beniazza, R.; Ibrahim, D.; Bose, G.; Robert, F.; Landais, Y. J. Org. Chem. 2009, 74, 6469.

http://dx.doi.org/10.1021/jo901395m

PMid:19655764

21. Lebeuf, R.; Robert, F.; Landais, Y. Org. Lett. 2005, 7, 4557.

http://dx.doi.org/10.1021/ol051377i

PMid:16209478

22. Dounay, A. B.; Overman, L. E. Chem. Rev. 2003, 103, 2945.

http://dx.doi.org/10.1021/cr020039h

PMid:12914487

23. Lodewyk, M. W.; Siebert, M. R.; Tantillo, D. J. Chem. Rev. 2012, 112, 1839. Scaling factors and methods were taken from the tables in the supporting information.

http://dx.doi.org/10.1021/cr200106v

PMid:22091891

24. Grigg, R.; Rasul, R.; Redpath, J.; Wilson, D. Tetrahedron Lett. 1996, 37, 4609. http://dx.doi.org/10.1016/0040-4039(96)00889-1

25. Celik, M.; Alp, C.; Coskun, B.; Gültekin, M. S.; Balci, M. Tetrahedron Lett. 2006, 47, 3659. http://dx.doi.org/10.1016/j.tetlet.2006.03.137 